\title{
Producción más limpia como estrategia ambiental preventiva en el proceso de elaboración de pasta de cacao. Un caso en la Amazonia Ecuatoriana
}

Carla Stephany Molina-Cedeño Briyidt Maryeli Pillco-Herrera ${ }^{2}$ EdisOn FABRICIO SALAZAR-MuÑOZ ${ }^{3}$ Billy DANIEl CORONEL-ESPINOZA ${ }^{4}$ Liliana Bárbara SarduY-Pereira ${ }^{5}$ Karel DiÉGUEZ-SANTANA ${ }^{6}$

\section{RESUMEN}

El cacao, es uno de los cultivos de mayor importancia en Ecuador, destinado a la exportación para ser utilizado en la elaboración de chocolate en otros países. El objetivo de este trabajo fue analizar el desempeño ambiental y las potencialidades de aplicación de mecanismos de producciones más limpias (PML) en la Asociación de productores de cacao fino de aroma "Tsatsayaku". Las herramientas de PML utilizadas fueron la revisión ambiental inicial, ecomapas, y análisis del proceso. Los resultados muestran que la generación de mucilago y cascarilla son los principales problemas ambientales del proceso. Se propusieron y evaluaron 2 opciones de mejora para controlar y disminuir la contaminación del proceso. El análisis muestra una relación $B / C$ de 1.38 y el Valor Actual Neto es de $\$ 36,001.37$ con una Tasa Interna de Retorno de $249.29 \%$. Finalmente, la aplicación de prácticas y tecnologías enmarcadas en sistemas de PML puede optimizar la utilización de materias primas y reducir los contaminantes sólidos y líquidos generados.

Palabras clave: Cacao; análisis costo-beneficio; pequeña y mediana industria; proceso tecnológico; tecnología limpia.

Cleaner production as a PREVENTIVE ENVIRONMENTAL STRATEGY IN THE COCOA PASTE PRODUCTION PROCESS. A CASE IN THE ECUADORIAN AMAZON

\section{ABSTRACT}

Cocoa is one of the most important crops in Ecuador, destined for export to be used in making chocolate in other countries. The objective of this work was to analyze the environmental performance and the potentialities of applying cleaner production mechanisms (CP) in the Association of producers of fine aroma cocoa "Tsatsayaku". The CP tools used were the initial environmental review, ecomaps, and process analysis. The results show that mucilage and cocoa husk generation are the main environmental problems of the process. Two improvement options were proposed and evaluated to control and reduce process contamination. The analysis shows a B / C ratio of 1.38 and the Net Present Value is $\$ 36,001.37$ with an Internal Rate of Return of $249.29 \%$. Finally, the application of practices and technologies framed in CP systems can optimize the use of raw materials and reduce the solid and liquid contaminants generated.

Keywords: Cocoa; cost-benefit analysis; small and medium industry; technological process; clean technology.

\section{INTRODUCCIÓN}

El crecimiento poblacional ha aumentado la demanda de alimentos, por lo que la industria ha tenido que diversificarse e incrementar los valores de producción. De igual manera, grandes cantidades son desperdiciadas y muchos recursos naturales se han empleado para satisfacer las elevadas demandas del sobreconsumo, por lo que estos niveles de producción resultan en impactos ambientales significativos con generación de cantidades de residuos sólidos, materiales de empaque, residuos de aditivos y efluentes (Diéguez-Santana, Arteaga-Pérez, Casas Ledon, y Rodríguez Rico, 2013; Rahim y Raman, 2015).

En el caso específico, del procesamiento del cacao, la industria mundial también ha introducido al mercado una gran variedad y cantidad de productos, lo que genera ingresos económicos a todas las personas dedicadas a la actividad. Pero, como plantea Konstantas, Jeswani, Stamford, y Azapagic (2018) durante el procesamiento del cacao pueden surgir problemas que afecten el equilibrio del ambiente, pues requiere una considerable

Ingeniera Ambiental, Personal de apoyo proyecto de investigación, Carrera Ingeniería Ambiental, Facultad Ciencias de la Vida, Universidad Estatal Amazónica, Puyo, Pastaza, Ecuador. ORCID: https://orcid.org/0000-0002-1538-3898

E-mail: amb2015316@uea.edu.ec

2 Ingeniera Ambiental, Personal de apoyo proyecto de investigación, Carrera Ingeniería Ambiental, Facultad Ciencias de la Vida, Universidad Estatal Amazónica, Puyo, Pastaza, Ecuador. ORCID: https://orcid.org/0000-0002-6055-9848

E-mail: amb2015345@uea.edu.ec

3 Ingeniero Ambiental, Personal de apoyo proyecto de investigación, Carrera Ingeniería Ambiental, Facultad Ciencias de la Vida, Universidad Estatal Amazónica, Puyo, Pastaza, Ecuador. ORCID: https://orcid.org/0000-0002-1208-8076 E-mail: amb2015373@uea.edu.ec

4 Magister en Sistemas Integrados de Gestión, Calidad, Ambiente y Prevención de Riesgos Laborales. Docente-Investigador de la Carrera Ingeniería Ambiental, Facultad Ciencias de la Vida, Universidad Estatal Amazónica, Puyo, Pastaza, Ecuador. ORCID: https://orcid.org/0000-0003-2389-5751 E-mail: bcoronel@uea.edu.ec

5 Licenciada en Educación, Especialidad Historia-Filosofía. Docente en Unidad Educativa Fiscomisional Cristóbal Colón, Parroquia Shell, Cantón Mera, Pastaza, Ecuador. ORCID: https://orcid.org/0000-0002-7970-3838 E-mail: lilianasarduy79@gmail.com

6 Master en Ingeniería en Saneamiento Ambiental, Docente-Investigador de la Carrera Ingeniería Ambiental, Facultad Ciencias de la Vida, Universidad Estatal Amazónica, Puyo, Pastaza, Ecuador. ORCID: https://orcid.org/0000-0003-4064-0566 E-mail:kdieguez@uea.edu.ec 
demanda de energía, agua y se generan residuos como es el caso del mucílago (baba) del cacao resultado del proceso de fermentación, que pueden convertirse en un problema ambiental cuando la disposición es inadecuada.

El cacao es uno de los principales cultivos en Ecuador, ocupa el $12 \%$ de la superficie cultivada y proporciona empleo directo a aproximadamente el 4\% de la población activa del país (Neira, 2016). En el 2019, Ecuador tuvo una producción de 297067 toneladas e ingresos $\$ 763880386$, siendo la mayor parte exportado a otros países en granos (86\%), para la elaboración de chocolate y otros subproductos (MAG, 2019). Las provincias con mayor producción de cacao se localizan mayoritariamente en la región costa, obteniendo la mayor cantidad de superficie cosechada en las provincia de Los Ríos, Guayas, Manabí (Castro, Oliveira, Tortorella, Zandonai, y Soares, 2017). Aunque, en los últimos años, las provincias amazónicas han comenzado a tener una activa participación en el sector. Varias pequeñas y medianas industrias han comenzado en los últimos años a elaborar productos en base a cacao nacional y fino de aroma, con fines de comercializar en los mercados locales y exportar a otros países, principalmente de Europa. con el paso de los años se ha tecnificado y crecido en todos los ámbitos de producción, y la industria del cacao está representada dentro del sector manufacturero que tiene un papel importante en la actividad económica del Ecuador, y crea una gran fuente de empleos en forma directa e indirecta.

Producciones más limpias. Una breve revisión de literatura.

La Organización de las Naciones Unidas para el Desarrollo Industrial (ONUDI) y el Programa de las Naciones Unidas para el Medio Ambiente (PNUMA) definieron por primera vez en 1989, a la Producción más limpia (PML), como "La aplicación continua de una estrategia ambiental integrada a los procesos, productos y servicios para aumentar la eficiencia y reducir los riesgos para los humanos y el medio ambiente" (UNEP, 1990). Durante tres décadas, se ha aplicado como estrategia para aumentar el cumplimiento de las regulaciones ambientales, y hoy en día es aceptada en todo el mundo (Padda y Asim, 2019).

Las PML se considera como un enfoque voluntario que se centra en la gestión ambiental para la reducción de la contaminación. Involucra, cambios en los procesos tecnológicos, consumos de recursos y las prácticas de reducción de desechos. Aborda los problemas en la fuente, y utiliza un enfoque del ciclo de vida del producto. Además, permite reducir los riesgos ambientales y de salud; minimizar el daño ambiental emplear mejor recursos y energéticos, lo que puede aumentar la eficiencia de los procesos de producción (Fritzen-Gomes, 2020; UNEP, 2015).

Las prácticas y principios implementados de producción más limpia contribuyen al desarrollo sostenible y la innovación de productos, debido a múltiples beneficios e impactos principalmente en tres frentes (Matos et al., 2018). El primero se centra en la eliminación o reducción de residuos, efluentes y emisiones en la fuente, lo que implica cambios generales en el producto o proceso de la empresa, algunos como: limpieza, sustitución de materias primas, cambios tecnológicos (Denham, Howieson, Solah, y Biswas, 2015). El segundo, involucra reciclaje interno, acciones como la recuperación y reutilización de energía y materiales desperdiciados en el proceso de producción pueden ser algunas de ellas. Mientras, el tercero involucra reciclaje externo, por el cual otras compañías usan los desechos como materia prima para sus productos; y el reciclaje de residuos, efluentes y / o emisiones por ciclos biogénicos, que busca la reintegración de la materia orgánica al medio ambiente a través de los procesos naturales (biogeoquímicos) (Filho, Nunhes, y Oliveira, 2019).

Varios estudios han abordado los factores que afectan la producción más limpia en las industrias Li y Hamblin (2016) argumentan que es necesario promover la innovación orientada al proceso y construir una cultura respetuosa con el medio ambiente para ser más activos en los resultados de la producción más limpia a largo plazo. Según Luken y Navratil (2004), entre otros factores que respaldan la implementación de medidas de producción más limpia están los sistemas de gestión general y las herramientas de gestión ambiental específicas, como los sistemas de control de procesos o las auditorías ambientales, que presumiblemente son parte de la producción más limpia. También, incide el personal de trabajo de una empresa que se considera como el impulsor clave de la adopción de tecnologías de producción más limpia (Blackman y Kildegaard, 2010), de ahí la importancia de que todas las partes estén involucradas en el proceso de adopción de PML.

Otro de los factores clave de la producción más limpia es la regulación ambiental, la literatura ha remarcado que tienen un efecto significativo en el cumplimiento cuando se trata de productos exportables (Roy, 2012), puesto que muchos de los importadores, demandan opciones de PML, en los procesos, por ejemplo, las empresas que exportan 
sus productos a países europeos (como es nuestro caso), deben cumplir con estándares ambientales específicos. En Ecuador, desde la Constitución de la República del Ecuador, considera la protección ambiental como uno de los deberes primordiales del Estado, en el Capítulo Segundo, Sección Segunda indica que éste: "reconoce el derecho de la población a vivir en un ambiente sano y ecológicamente equilibrado, que garantice la sostenibilidad y el buen vivir, sumak kawsay". Mientras, el artículo 15, menciona que "El Estado promoverá en el sector público y privado, el uso de tecnologías ambientalmente limpias y energías alternativas no contaminantes y de bajo impacto" (Asamblea Constituyente, 2008). Por su parte, el Código Orgánico del Ambiente, el Artículo 245, del Título VI. Producción y consumo sustentable, y Libro 3 Calidad Ambiental, establece las obligaciones generales para la producción más limpia y el consumo sustentable, para todas las instituciones del Estado y las personas naturales o jurídicas, el literal 4, "Prevenir y minimizar la generación de cargas contaminantes al ambiente, considerando el ciclo de vida del producto"; mientras el literal 5 menciona "Fomentar procesos de mejoramiento continuo que disminuyan emisiones"; y el literal 9. "Minimizar y aprovechar los desechos", por lo que estrategias encaminadas a ello, se encuentran amparadas bajo el marco normativo nacional (Asamblea Nacional, 2017).

En 1995, la ONUDI y el PNUMA establecieron los primeros Centros Nacionales de Producción más Limpia en ocho países, para la promoción e incorporación de políticas de PML en los sectores industriales (Luken, Van Berkel, Leuenberger, y Schwager, 2016). Mientras, que, en Ecuador, en el año 2013 surgió el Centro Ecuatoriano de Eficiencia de Recursos y Producción más Limpia (CEER), para apoyar esa actividad a nivel nacional (CEER, 2016). Recientemente, estudios realizados a nivel nacional han abordado algunos sectores productivos, como el aprovechamiento de residuos de mataderos de animales (Caiza, Chimbo, Sarduy-Pereira, Pisco, y Diéguez-Santana, 2018), o estrategias de PML evaluadas para granjas de animales (Cárdenas, Maldonado, Valdez, Sarduy-Pereira, y Diéguez-Santana, 2019). Sin embargo, varios sectores importantes no han sido estudiados, por lo que la colocación de las PML, como enfoque preventivo en su desempeño, puede incrementar la generación de ingresos y beneficios ambientales.

Según la revisión de la literatura, las producciones más limpias abarcan desde los aspectos normativos, hasta los criterios productivos y económicos. Además, en comparación con otros sectores, las industrias procesadoras de cacao no han recibido tanta atención y existen estudios de casos limitados sobre la implementación de estrategias de PML. Por otra parte, las PML, a pesar de ser ampliamente difundida, sigue siendo muy importante en términos técnico, económicos y ambientales para las pequeñas y medianas empresas, principalmente en países en vías de desarrollo. Dado que el sector del cacao es un rubro importante en la economía y en Ecuador existen a pequeña y mediana escala instalaciones de procesamiento, este estudio se realizó con el objetivo de demostrar que la estrategia de PML puede ayudar a la industria de procesamiento del cacao a mejorar su desempeño ambiental y económico. Además, puede contribuir a comprender como las opciones de PML, pueden diversificar las actividades productivas, que, en la región amazónica, con las limitaciones de medios de vida, puede generar empleos e ingresos económicos.

En el ámbito de las PML, estas empresas no han hecho intentos en aplicar Producción Más Limpia y de esta manera contribuir al cuidado del medio ambiente. Específicamente, los pequeños productores de cacao son los que presentan mayores dificultades en la aplicación de las PML, pues la mayoría de estas empresas no están ubicadas en los Parques Industriales y deben gestionar sus propios residuos. Las instalaciones de la Asociación se encuentran ubicadas en un área rural y presenta los problemas frecuentes de la pequeña y mediana industria, que van desde la falta de capacitación y conocimientos sobre aspectos ambientales hasta déficit de información sobre los tipos de contaminantes generados y potencialidades de aprovechamiento in situ de los mismos (Arteaga-Pérez, Segura, y Santana, 2016).

El estudio de las estrategias de Producción más limpia a implementar en el proceso de elaboración de pasta de cacao es de gran importancia para considerar como caso de estudio, pues en la región amazónica en los últimos años han comenzado a surgir emprendimientos en el sector productivo y no existen reportes de aplicación de PML en la producción de cacao. Por otra parte, la información de este documento también será útil para otras empresas del sector, incluso para la pequeña y mediana empresa nacional que necesita reactivación y mejor desempeño ambiental. Los criterios identificados en el diagnostico pueden ser empleados para evaluar el estado actual del proceso en la asociación y ser una línea de base con miras a implementar escenarios futuros de optimización de la empresa. Además, aporta nueva información sobre estrategias de aprovechamiento de residuos para generar nuevos productos que pueden ser a futuro 
incorporados en cadenas de comercialización. Debido a la importancia de la viabilidad de implementar las estrategias que se discuten, este documento busca definir criterios para seleccionar cuales pueden ser más viables en orden de beneficios, ambientales, como mitigación de la contaminación, y productivo-económicos, como mejora del proceso en cuanto a productividad, eficiencia del producto y nuevas oportunidades de negocio con los nuevos productos.

Problema: ¿Cuáles son las opciones de PML, que pueden mejorar el proceso productivo del centro de producción de la Asociación de productores de cacao fino de aroma?

\section{Este documento tiene como objetivos:}

- Diagnosticar el estado actual de producción de barras de cacao de la Asociación de productores de cacao fino de aroma "Tsatsayaku" del Cantón Carlos Julio Arosemena Tola.

- Identificar las principales opciones de producción más limpia en la cadena productiva de cacao fino de aroma.

- Evaluar la viabilidad técnica, económica y ambiental de las opciones propuestas para minimizar los impactos generados en la producción de barras de cacao de la mencionada Asociación.

Hipótesis: Si se aplican las opciones de producciones más limpias mejorarán los procesos y control de la contaminación de la cadena productiva de cacao fino de aroma, de la Asociación de productores "Tsatsayaku" del Cantón Carlos Julio Arosemena Tola.

\section{MATERIALES Y MÉTODOS}

\subsection{Localización}

El estudio se llevó a cabo en la provincia de Napo, en el cantón Carlos Julio Arosemena Tola; Comunidad Nueva Esperanza, en los predios de la Asociación de productores de cacao Tsatsayaku. La planta de producción de la asociación cuenta con una extensión de $10000 \mathrm{~m}^{2}$, con coordenadas geográficas 185385.29 m E - 9874891.02 m S.

\subsection{Criterios metodológicos del análisis de PML}

La investigación se dividió en 5 etapas, donde se analizaron criterios del Programa de PML de la ONUDI, además de las guías nacionales de PML para Bolivia (CPTS, 2005) y para Ecuador (CEER,
2019). Adicionalmente se revisaron casos publicados en Ecuador (Caiza et al., 2018; Cárdenas et al., 2019; Guallo-Aguinda et al., 2020).

Etapa I: Organización y definición de objetivos y metas de PML: Esta primera etapa, se inició con la socialización a la asociación para exponer los beneficios de la aplicación de las PML. Además, se organizó el equipo, se definieron las funciones y se identificaron los obstáculos y barreras. También se definieron los conceptos sobre las PML, y se revisó la legislación ambiental aplicable en el sector a nivel nacional.

Etapa II: Diagnóstico técnico, económico y ambiental preliminar de la empresa o proceso: En este sección se recopiló información de producción, insumos, costos y otros datos del proceso. Se realizaron entrevistas a personal clave (propietarios y trabajadores). Adicionalmente, se obtuvo información ambiental de los principales impactos negativos generados por la actividad. Se confeccionó un ecomapa con los criterios ambientales más significativos por cada etapa, y con la finalidad de visualizar cada proceso desarrollado en la planta de procesamiento.

Etapa III: Evaluación técnico, económico y ambiental de la empresa: Se elaboraron los balances de materiales de las operaciones unitarias involucradas. Para ello, se cuantificaron todas las materias primas empleadas, insumos y energía, subproductos generados, corrientes de residuos sólidos, efluentes líquidos, y emisiones. Se identificaron las causas de las ineficiencias y se seleccionaron las oportunidades a ser evaluadas en términos técnicos y económicos (CEER, 2019).

Etapa IV. Formulación de alternativas de producción más limpia: Una vez propuestas las opciones de PML, fue necesario evaluar su potencial implementación. Para ello, se realizó la evaluación de la factibilidad técnica, económica y ambiental, a fin de seleccionar las más factibles. Además, se analizó las variación de los indicadores del proceso.

Etapa V. Implementación, Monitoreo y control: Esta última etapa incluye cuales son los requerimientos para implementar las alternativas de PML definidas, previamente. En este caso es necesario considerar un plan de acción con los responsables, presupuesto para cada actividad plazos o cronograma, e indicadores de seguimiento. Mientras, como parte, del monitoreo y control, se evalúa los indicadores técnico, económicos y ambientales de la actividad. 


\subsubsection{Etapa I}

\section{a. Descripción de la empresa. Estructura orga- nizacional}

La Asociación de productores de cacao fino de aroma "Tsatsayaku", está registrada en la Superintendencia de Economía Popular y Solidaria desde el 05 de abril del 2013. Tiene el apoyo de algunas instituciones públicas y privadas, Gobiernos Municipales, Provinciales y ONGs, el Programa de Pequeñas Donaciones de las Naciones Unidas, el Ministerio de Agricultura y Ganadería, y la cooperativa Maquita Cushunchic, son algunas de ellas. Sus principales metas son la producción, comercialización e industrialización del cacao fino de aroma y sus derivados, con estándares internacionales de calidad, y tecnologías amigables con el medio ambiente. Sus instalaciones cuentan con una infraestructura que incluye un centro de acopio ubicado en la comunidad Nueva Esperanza, con una capacidad instalada para almacenar un estimado de 2000 quintales de cacao seco en el año (Mancheno, 2018). El principal producto que comercializa desde el 2015 en mercados nacionales e internacionales es pasta de fino cacao, bajo la marca Tsatsayaku, en diferentes presentaciones.

\section{Organización}

La Asociación está compuesta por familias kichwas y colonas, que se dedican principalmente a los cultivos de cacao, guayusa y otros alimentos a partir de la chakra. Como estructura están organizados por 180 productores de 13 comunidades locales. Un aspecto importante de la Asociación es su rol en la gobernanza ambiental, pues desde los inicios de su creación ha sobresalido en el territorio, teniendo una participación activa en eventos como la Ruta del Cacao, actualmente la Mesa de Chakra. El organigrama general de la empresa abarca desde nivel directivo (Junta general de socios), con un administrador, asesor jurídico, secretaria-contadora, hasta nivel operativo con tres trabajadores.

\subsubsection{Etapa II. Diagnóstico técnico - económico y ambiental preliminar de la empresa}

\section{a. Descripción del proceso}

La producción de chocolate al 100\% puro, Nibs, y sus derivados se hace 2 veces por semana debido a que en el proceso de refinado se demora de 18 a 24 horas. En la Figura 1 se muestra cada una de las etapas que se trabajan dentro del procesamiento del cacao para la obtención de chocolate.

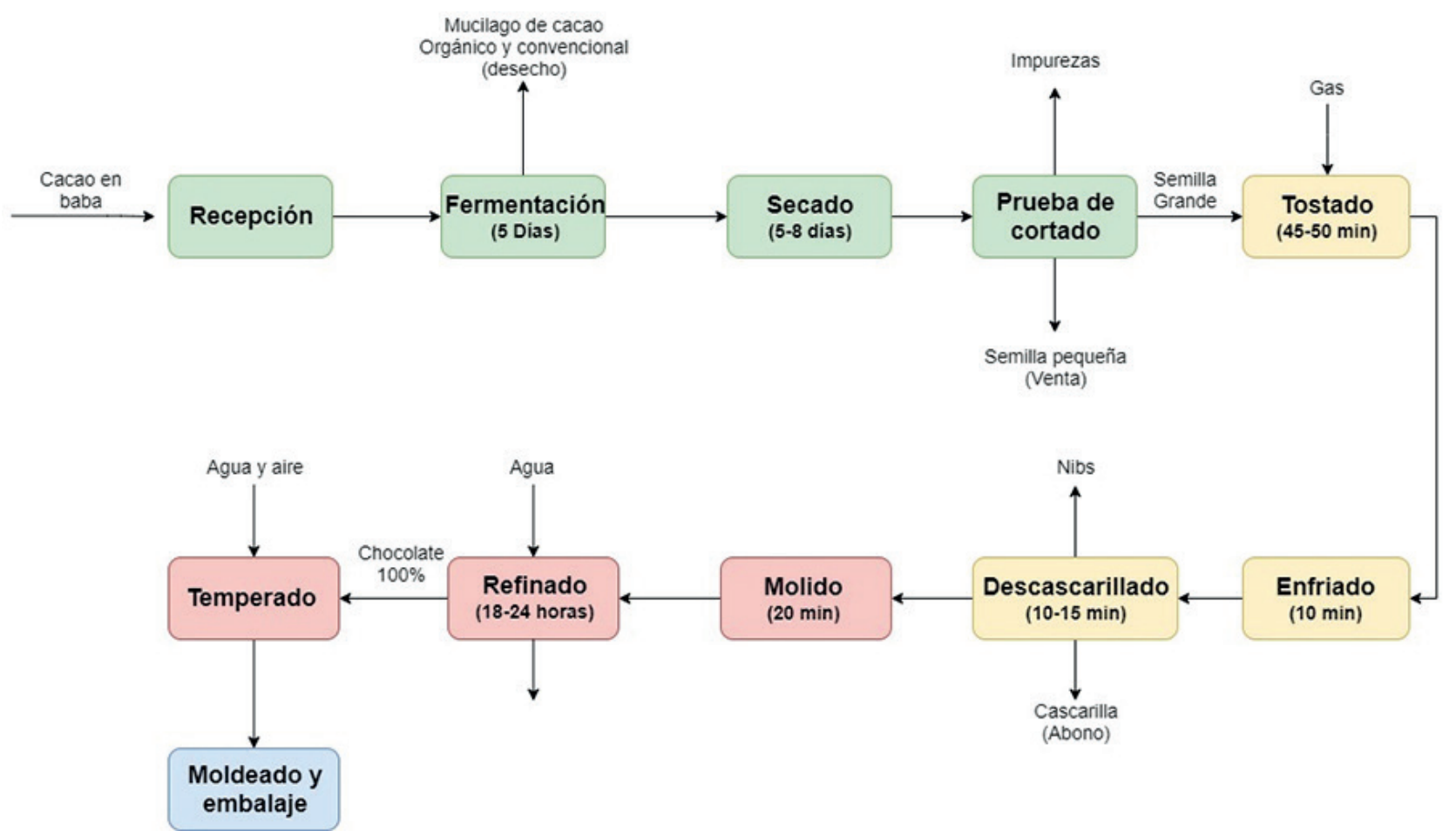

Figura 1. Diagrama de procesos para la obtención de chocolate.

Fuente: Elaboración propia. 
1. Recepción: El cacao es comprado a los agricultores socios en la temporada de cosecha (enero a julio), receptando aproximadamente 279 Quintales de 3 tipos de Cacao, orgánico (98 Quintales), Convencional (176 quintales) y Cacao CCN51 (5 quintales) con un valor de $\$ 0.40$ / lb, $\$ 0.35 / \mathrm{lb}$ y $\$ 0.30$ / lb, respectivamente.

2. Fermentación: El cacao fresco pasa un proceso de fermentación en cajas madera (4 quintales por caja) durante aproximadamente 5 días, aquí se vigilan las características organolépticas (aroma, sabor, textura) del cacao. El mucílago o baba del cacao convencional y orgánico pasan a ser almacenados en un pozo sin tratamiento, mientas que el mucílago del cacao CCN51 se aprovecha para la elaboración de helados.

3. Secado: Esta actividad se realiza en marquesinas aprovechando el calor del sol por lo que puede durar entre 5 a 8 días hasta que las habas de cacao se sequen dependiendo de las condiciones climáticas de la zona. El cacao CCN51 se vende en pepa y no pasa a procesamiento de chocolate, por otro lado, el cacao orgánico y convencional pasa al siguiente proceso. Finalmente, las habas salen con una humedad menor al $7 \%$.

4. Prueba de corte: Aquí las habas de cacao deben tener del 70 a $85 \%$ de fermentación; las habas pequeñas se venden a un valor entre $\$ 1$ y $\$ 1.10$, mientras que las habas grandes pasan al proceso de chocolate. Una vez seleccionado el cacao de calidad para el procesamiento de chocolate continúan los siguientes procesos que se realizan 3 veces por día:

5. Tostado: Las habas de cacao ingresan a la tostadora, que trabaja con gas y un motor a $110 \mathrm{v}$, el proceso dura aproximadamente de 45 a 50 min con una temperatura que va desde los 80 a $95^{\circ} \mathrm{C}$.

6. Enfriado: En esta etapa se adiciona aire a temperatura ambiente mediante una bomba centrífuga y en un tiempo inferior a $10 \mathrm{~min}$.

7. Descascarillado: Las habas una vez tostadas y enfriadas pasan a la descascarilladora, aquí se obtiene como subproductos los Nibs que son trocitos de semillas de cacao tostado y las cascarillas que se venden como abono a $\$ 10$ el quintal, por cada quintal de cacao se obtiene el $5 \%$ de cascarilla.
8. Molido: Una vez tostadas y descascarilladas las habas son molidas de nuevo este proceso dura un 20 min aproximadamente.

9. Refinado: En esta etapa se busca reducir el tamaño de la partícula del cacao quedando con un micraje de 18 a 20, este es el proceso más largo ya que dura de 18 a 24 horas y se trabaja con una olla de doble fondo con una capacidad de $35 \mathrm{~kg}$ de licor de cacao y aproximadamente $0,078 \mathrm{~m}^{3}$ de agua a una temperatura de $45^{\circ} \mathrm{C}$ con unas aspas circulares de acero inoxidable. En este proceso se puede obtener licor de cacao al $100 \%$ de pureza.

10. Temperado: Esta parte del proceso, las temperaturas fluctúan de $50^{\circ} \mathrm{C}$ a $26^{\circ} \mathrm{C}$ y luego a $32^{\circ} \mathrm{C}$ al igual que el anterior proceso se trabaja con una olla de doble fondo en la que no solo se suministra agua si no también aire.

11. Moldeado y embalaje: El moldeado se lo realiza en una mesa de acero inoxidable con moldes plásticos, en el caso del licor de cacao al 50\% se mezcla con maní y se manda a los moldes para luego pasar a ser empacados y etiquetados en presentaciones pequeñas de 200 gramos, por otro lado el licor de cacao al $100 \%$ que se distribuye localmente se empaca en presentaciones de 250 y 450 gramos, mientras el que se exporta a Inglaterra se empaca en plásticos y cartones por kilogramo; todo esto se realiza en un cuarto frio a $17^{\circ} \mathrm{C}$.

\section{b. Resultados del diagnóstico de Producción Más Limpia}

La revisión ambiental inicial permitió identificar los principales problemas ambientales que presenta la fabricación de licor de cacao al $100 \%$, estos son: la inadecuada disposición del mucílago del cacao resultante del proceso de fermentación, la generación de agua residual producto del lavado de los equipos y maquinarias, la generación de residuos sólidos como plástico y cartón, en el área de envasado, empaquetado y etiquetado, además de los residuos de cascarilla producto del área de descascarillado y finalmente el alto consumo energético en todo el sistema de producción. Estos problemas son frecuentes de la actividad, pues según Recanati, Marveggio, y Dotelli (2018) en su estudio de la evaluación del ciclo de vida desde la semilla hasta la barra de chocolate en una cadena de suministro de chocolate sostenible, la fabricación del chocolate genera aguas residuales y diferentes tipos residuos sólidos industriales, además de que existe 
un alto consumo energético con relación al tipo de maquinaria y el tiempo que se utiliza.

Con parte de la información obtenida, se elaboró el ecomapa. La figura 2 muestra, la distribución de las áreas de la planta de producción, equipos utilizados y las principales corrientes contaminantes observadas in-situ como: consumo energético y la utilización de gas de consumo doméstico, la generación de agua residual y la disposición final del mucílago.

\subsubsection{Etapa III: Evaluación técnico, económico y ambiental de la empresa}

La Tabla 1 muestra las entradas y salidas de las 11 etapas expuestas en la descripción del proceso. Como se puede observar a partir de los 45,67 Quintales de cacao en baba que se procesan al mes se obtiene 2,74 quintales de licor de cacao esto a causa de que existen pérdidas en los procesos como: fermentación (2\%), prueba de corte $(60 \%)$, tostado $(4 \%)$, descascarillado $(5 \%)$, y molido, refinado, temperado ( $1 \%$ pérdida en equipo), además de que se obtiene subproductos como los nibs que representan 2,61 quintales. Por otra parte, la tabla 2 muestra el consumo mensual de los energéticos, donde se consume un cilindro de gas licuado de petróleo (GLP), equivalente a $15 \mathrm{~kg}$, debido a que solo se ocupa en el proceso de tostado, el consumo energético que representa el uso de los equipos es de $772,26 \mathrm{~kW} / \mathrm{mes}$ y el consumo de agua tomado en cuenta en el proceso de refina y temperado es de $864.36 \mathrm{~L} / \mathrm{mes}$. Estos valores son acordes al proceso normal de obtención del chocolate, como menciona Vásquez et al. (2019) en la revisión de enfoques biotecnológicos para la gestión de residuos de cacao, aproximadamente el $80 \%$ de la fruta de cacao se descarta como biomasa residual, incluidas las cáscaras de vaina de cacao, las cáscaras de grano de cacao y los sudores de cacao (Mucílago).

Mientras, la Tabla 2 muestra los flujos de agua, electricidad y GLP, por cada etapa del proceso.

La tabla 3, muestra las materias primas, insumos, energéticos, mano de obra, y otros gastos necesarios para un ciclo productivo mensual.

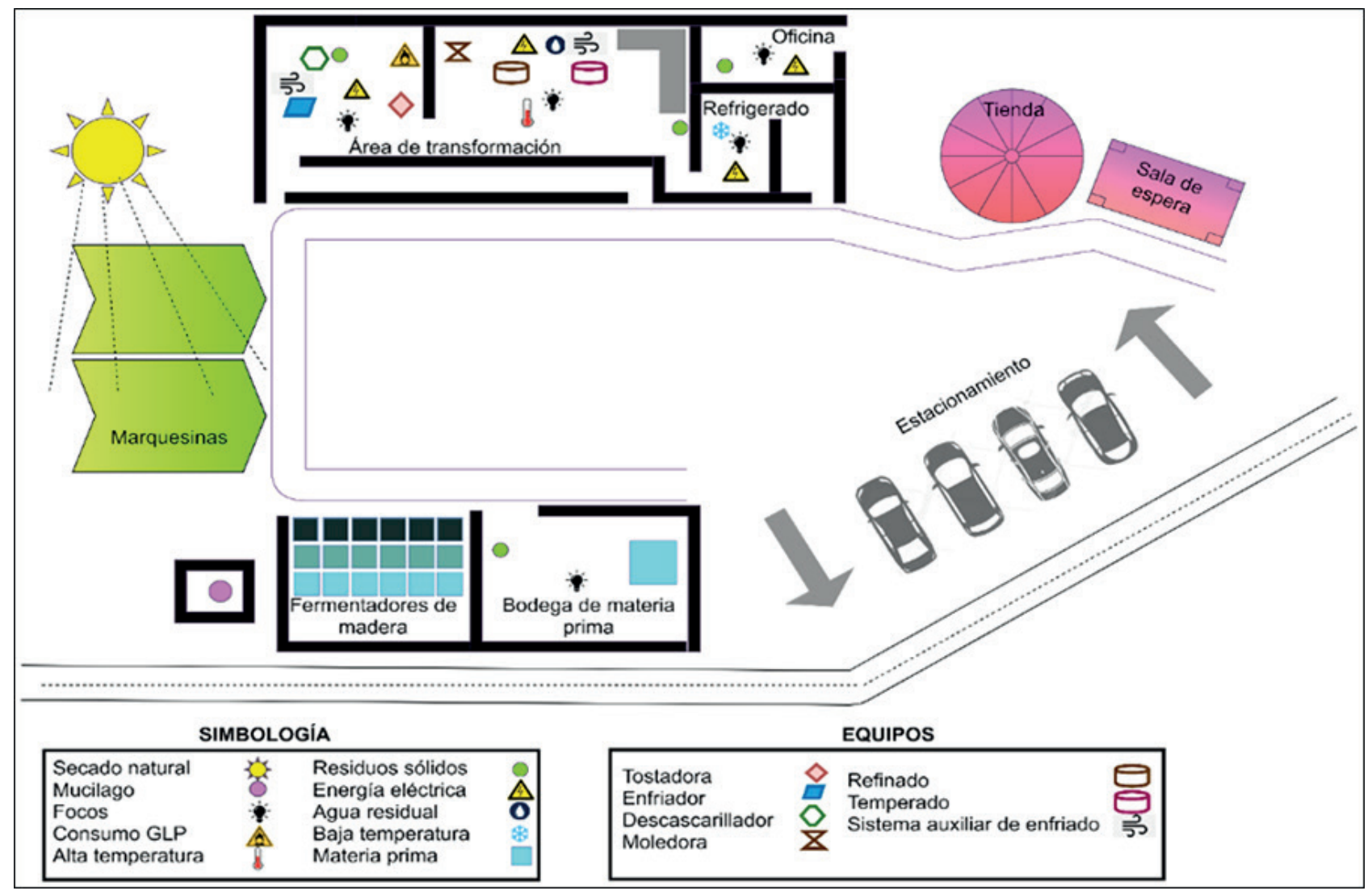

Figura 2. Ecomapa de la planta de procesamiento de cacao de la asociación Tsatsayaku. 
Tabla 1. Análisis de flujo de la materia prima, por mes

\begin{tabular}{|c|c|c|c|c|}
\hline Etapa & $\begin{array}{c}\text { Tipo de materia } \\
\text { entrada }\end{array}$ & Cantidad, kg & Tipo de materia entrada & Cantidad, kg \\
\hline Recepción & Cacao fresco & 4576.6 & Caca fresco & 4576.6 \\
\hline \multirow{2}{*}{ Fermentación } & Cacao fresco & 4576.6 & Cacao fermentado & 4485.4 \\
\hline & & & Mucilago & 91.19 \\
\hline \multirow{2}{*}{ Secado } & Cacao fermentado & 4485.4 & Cacao seco & 1495.13 \\
\hline & & & Agua Evaporada & 2990.27 \\
\hline \multirow{2}{*}{ Prueba Corte (Selección) } & Cacao seco & 1495.13 & Cacao seleccionado & 598.25 \\
\hline & & & Rechazo+Impurezas & 896.88 \\
\hline \multirow{2}{*}{ Tostado } & Cacao seleccionado & 598.25 & Cacao tostado & 574.2 \\
\hline & & & Cacao quemado, cenizas & 24.05 \\
\hline Enfriado & Cacao tostado & 574.2 & Cacao tostado & 574.2 \\
\hline \multirow{3}{*}{ Descarrillado } & Cacao tostado & 574.2 & Cacao descascarillado & 283.6 \\
\hline & & & Nibs & 261.55 \\
\hline & & & Cascarilla & 29.05 \\
\hline \multirow{2}{*}{ Molido } & Cacao descascarillado & 283.6 & Cacao molido & 280.76 \\
\hline & & & $1 \%$ pérdidas equipo & 2.84 \\
\hline \multirow{2}{*}{ Refinado } & Cacao molido & 280.76 & Cacao refinado & 277.95 \\
\hline & & & $1 \%$ pérdidas equipo & 2.81 \\
\hline \multirow{2}{*}{ Temperado } & Cacao refinado & 277.95 & Cacao temperado & 275.17 \\
\hline & & & $1 \%$ pérdidas equipo & 2.78 \\
\hline \multirow{2}{*}{ Envasado y etiqueta } & Cacao temperado & 275.17 & Cacao envasado & 273.8 \\
\hline & & & $0.5 \%$ pérdidas envase & 1.38 \\
\hline
\end{tabular}

Fuente: Elaboración propia.

Tabla 2. Análisis de flujo agua e insumos energéticos por mes

\begin{tabular}{|l|c|c|c|}
\hline \multicolumn{1}{|c|}{ Etapa } & Consumo Agua, L & Consumo Electricidad, kWh & Consumo GLP, kg \\
\hline Tostado & & 13.64 & 15 \\
\hline Enfriado & & 1.52 & \\
\hline Descarrillado & & 1.52 & \\
\hline Molido & & 1.98 & \\
\hline Refinado & & 262.4 & \\
\hline Temperado & 626.32 & 11.2 & \\
\hline Envasado y etiqueta & 243.04 & 480 & \\
\hline
\end{tabular}

Fuente: Elaboración propia.

Basado en estos valores, la ganancia mensual de $\$ 1140.44$, con valores de la tasa beneficio/costo de 1.20 , es decir que la asociación percibe $\$ 0.20$ de ganancia por cada dólar que se invierte en el proceso.

\subsubsection{Etapa IV. Formulación de alternativas de producción más limpia:}

A partir de los resultados del análisis del proceso de la planta de producción de chocolate, se identificaron problemas y se propusieron opciones de
PML, para revalorizar subproductos como el mucílago y la cascarilla del procesamiento del cacao. Estas fueron: Elaboración de un herbicida natural a partir del mucílago de cacao y elaboración de té de cascarilla en combinación con guayusa. Según Arteaga (2013) los principales factores por los que no se aprovecha estos residuos es por falta de conocimiento de los agricultores sobre el grado de nutrientes, azúcares, fibras y proteínas que contienen, ya que la mayoría se enfocan al aprovechamiento del grano y consideran a la cáscara de cacao 
Tabla 3. Balance de materias primas, insumos, consumos energéticos, gastos mensuales del proceso.

\begin{tabular}{|c|c|c|c|c|}
\hline \multicolumn{5}{|c|}{ Consumos, gastos, productos (1 ciclo mensual) } \\
\hline Rubro & $\begin{array}{l}\text { Unidad medida } \\
\text { Entradas }\end{array}$ & Cantidad & Costo unitario, $\$$ & Costo Total, \$ \\
\hline \multicolumn{5}{|l|}{ Materia Prima e Insumos } \\
\hline Cacao Orgánico & $\mathrm{lb}$ & 3600.1 & 0.4 & 1440.0 \\
\hline Cacao convencional & $\mathrm{lb}$ & 6466.1 & 0.35 & 2263.1 \\
\hline Cartón & $U$ & 10 & 1.1 & 11 \\
\hline Gas & Cilindro $15 \mathrm{~kg}$ & 1 & 3.5 & 3.5 \\
\hline Fundas $(250 \mathrm{~g})$ & $U$ & 250 & 0.08 & 20 \\
\hline Fundas (450 g) & $U$ & 130 & 0.1 & 13 \\
\hline Fundas (Nibs) & $\mathrm{U}$ & 1100 & 0.13 & 143 \\
\hline Plástico embalaje & $\mathrm{m}$ & 3 & 0.5 & 1.5 \\
\hline Etiquetas/sellos & Unidad & 1490 & 0.03 & 44.7 \\
\hline Subtotal & & & & 3939.9 \\
\hline \multicolumn{5}{|c|}{ Consumo energía eléctrica y agua } \\
\hline Electricidad & kwh & 1000 & 0.13 & 130 \\
\hline Agua & $\mathrm{m}^{3}$ & 150 & 0.5 & 75 \\
\hline Subtotal & & & & 205 \\
\hline \multicolumn{5}{|c|}{ Talento humano planta } \\
\hline Trabajadores & Salario/mes & 3 & 394 & 1182 \\
\hline Seguro trabajadores y Aporte IESS & Salario/mes & 3 & 77.25 & 231.75 \\
\hline Subtotal & & & & 1413.75 \\
\hline \multicolumn{5}{|c|}{ Servicios y otros gastos } \\
\hline Mantenimiento & $U$ & 1 & 48.33 & 48.33 \\
\hline Subtotal & & & & 48.33 \\
\hline Total egresos & & & & 5606.96 \\
\hline \multicolumn{5}{|c|}{ Productos terminados } \\
\hline Barra de chocolate $250 \mathrm{gr}$ & Barra & 211 & 4.5 & 949.5 \\
\hline Barra de chocolate $450 \mathrm{gr}$ & Barra & 125 & 7 & 875 \\
\hline Cubo de chocolate $1500 \mathrm{gr}$ & Caja & 110 & 21 & 2310 \\
\hline Nibs 250gr & Funda & 1044 & 2.5 & 2610 \\
\hline Cascarilla (abono) & qq & 0.29 & 10 & 2.9 \\
\hline Total Ingresos & & & & 6747.4 \\
\hline
\end{tabular}

Fuente: Elaboración propia.

y mucílago como materia inservible, así también (Vásquez et al., 2019) afirma que los agricultores descartan alrededor del $80 \%$ de la fruta de cacao como biomasa residual generalmente en los primeros pasos de procesamiento de cacao, y que por su inadecuada eliminación ha suscitado preocupaciones sociales y ambientales.

En cuanto a las aguas residuales, en el proceso productivo, se vierten directamente al suelo del lugar y los diferentes cuerpos hídricos. Estas descargas pueden incidir en problemas ambientales como la acidificación y eutrofización, debido a la presencia de nitrógeno y fósforo principalmente. (Diéguez-Santana,
Casas-Ledón, Loureiro-Salabarria, Pérez-Martínez, y Arteaga-Pérez, 2020; Soto-Cabrera, Panimboza-Ojeda, Ilibay-Granda, Valverde-Lara, y Diéguez-Santana, 2020). Aunque, resultados del monitoreo, presentaron que cumplían con los parámetros establecidos en el Acuerdo Ministerial 097-A, de descargas a cuerpos de agua dulce.

\section{a. Descripción de opciones}

Herbicida natural a partir del mucilago de cacao: Este herbicida natural será empleado para el control de malezas en cultivos de ciclo corto, e intenta promover como plantea (Sanchez, Perez, Rojas, y Trujillo, 2019) la disminución de herbicidas químicos 
y mejorando el equilibrio del medio ambiente. Para la elaboración del herbicida se ha determinado una relación de $50 \%$ de mucílago con $50 \%$ de agua que logra mayores promedios de mortalidad de malezas a los 8 y 15 días.

Té de cascarilla en combinación con guayusa: la elaboración de té a base de cascarilla y guayusa que al tener de un 2 a $3 \%$ de cafeína ayuda a elevar la fuerza de una persona, que en términos médicos se lo denomina como un tónico y estimulante, así también Okiyama, Navarro, y Rodrigues (2017) asegura que, por su alto contenido de compuestos fenólicos, tiene una aplicación potencial como agente antioxidante. El té se elabora mediante una combinación de un $80 \%$ de cascarilla de cacao con un $20 \%$ de guayusa, que, según Llerena, Ah-Hen, y Lemus-Mondaca (2017) tiene una mayor aceptabilidad por los degustadores.

\section{b. Viabilidad técnico-económica y ambiental del proceso y de las opciones de produccio- nes más limpias}

La tabla 4, muestra las materias primas, insumos, energéticos, mano de obra, y otros gastos empleados para elaborar las dos propuestas formuladas.

Como se puede apreciar, se gastan $\$ 1433.19$, dado principalmente por las materias primas e insumos necesarios para la elaboración del té, y la mano de obra adicional, pero aun con estos gastos los ingresos de $\$ 2904.5$ son superiores y reportan ganancias a la asociación. A partir de estos datos se puede realizar el análisis económico para determinar su viabilidad al implementarlas dentro del proceso de la obtención de chocolate actual. Considerando el proceso sin PML y con las opciones de PML, el balance se muestra en la Figura 3. Con la implementación de las estrategias de producción

Tabla 4. Materias primas, insumos, consumos energéticos, gastos e ingresos de las propuestas de PML

\begin{tabular}{|c|c|c|c|c|}
\hline \multicolumn{5}{|c|}{ Materia Prima e Insumos } \\
\hline Rubro & Unidad medida & Cantidad & Costo unitario, $\$$ & Costo Total, \$ \\
\hline Bolsa de Té & Unidad & 10550 & 0.05 & 527.5 \\
\hline Cajas para té & Unidad & 705 & 0.4 & 282 \\
\hline Guayusa & $\mathrm{g}$ & 5800 & 0.02 & 116 \\
\hline Gas & Cilindro $15 \mathrm{~kg}$ & 1 & 3.5 & \\
\hline Envases, $1 \mathrm{~L}$ & Unidad & 7 & 0.17 & 1.19 \\
\hline Envases, 5L & Unidad & 6 & 0.5 & 3 \\
\hline Subtotal & & & & 933.19 \\
\hline \multicolumn{5}{|l|}{ Depreciación de equipos } \\
\hline Tanque plástico $200 \mathrm{~L}$ & 1 & & 3.25 & 3.25 \\
\hline Subtotal & & & & 3.25 \\
\hline \multicolumn{5}{|l|}{ Consumo energía eléctrica y aguas } \\
\hline Electricidad & kwh & 100 & 0.13 & 13 \\
\hline Agua & $\mathrm{m}^{3}$ & 25 & 0.5 & 12.5 \\
\hline Subtotal & & & & 25.5 \\
\hline \multicolumn{5}{|l|}{ Talento humano planta } \\
\hline Trabajadores & Salario/mes & 1 & 394 & 394 \\
\hline Seguro trabajadores y Aporte IESS & Salario/mes & 1 & 77.25 & 77.25 \\
\hline Subtotal & & & & 471.25 \\
\hline Total de egresos & & & & 1433.19 \\
\hline \multicolumn{5}{|c|}{ Ingresos o productos nuevos } \\
\hline Herbicida de mucílago de cacao, $1 \mathrm{~L}$ & $U$ & 7 & 2.5 & 17.5 \\
\hline Herbicida de mucílago de cacao, $5 \mathrm{~L}$ & $U$ & 6 & 12.5 & 75 \\
\hline Té de cascarilla de cacao y guayusa & Caja & 703 & 4 & 2812 \\
\hline Total de ingresos & & & & 2904.5 \\
\hline
\end{tabular}

Fuente: Elaboración propia. 
más limpias mencionadas con anterioridad se reflejan cambios económicos positivos en la empresa, pues se incrementan las ganancias mensuales a \$2632.96 dólares (frente a los \$1140.44 sin las opciones), y además la relación beneficio/costo de la asociación se incrementa considerablemente hasta 1.38 , obteniendo $\$ 0.18$ más de ganancias que en el estado actual, recalcando que para la implementación de las estrategias propuestas no se requiere la adquisición de maquinarias y equipos, puesto que ya se cuenta con las mismas. Adicionalmente, la aplicación de las opciones tiene un Valor Actual Neto (VAN) de $\$ 36,001.37$ y una Tasa Interna de Retorno (TIR) de 249.29\%.

\section{c. Viabilidad ambiental del proceso. Compara- ción del proceso actual, con las opciones de producciones más limpias}

Contrastación de la hipótesis de la investigación.

Finalmente, una vez evaluadas técnico y económicamente las opciones se contrastó, la hipótesis de la investigación y se realizó la comparación del proceso, en base a dos alternativas:
1. Proceso actual sin considerar opciones PML

2. Proceso con la aplicación de opciones de PML

Si se muestran cambios en los indicadores ambientales del proceso en orden positivo, entonces se cumple que mejorarán los procesos y el control de la contaminación con la aplicación de las opciones de PML propuestas para el proceso de producción de cacao de la Asociación. Estos cambios implicaron varias propuestas de prácticas y tecnologías enmarcadas en las PML, tales como: ahorro de electricidad, en los sistemas de climatización, optimización del tiempo de trabajo innecesario y mejor empleo de la iluminación natural de la instalación. Otro recurso, considerado fue el agua, mediante una propuesta de ahorro de agua, principalmente de la limpieza del equipamiento e instalación. En cuanto a las opciones de aprovechamiento de subproductos se abordaron las dos propuestas, la cascarilla de cacao para la elaboración de té y la disminución de las cargas orgánicas de las aguas residuales mediante el aprovechamiento del mucílago. En este caso, la tabla 5 muestra los principales eco-indicadores representados en la base a $\mathrm{kg}$ de producto obtenido.

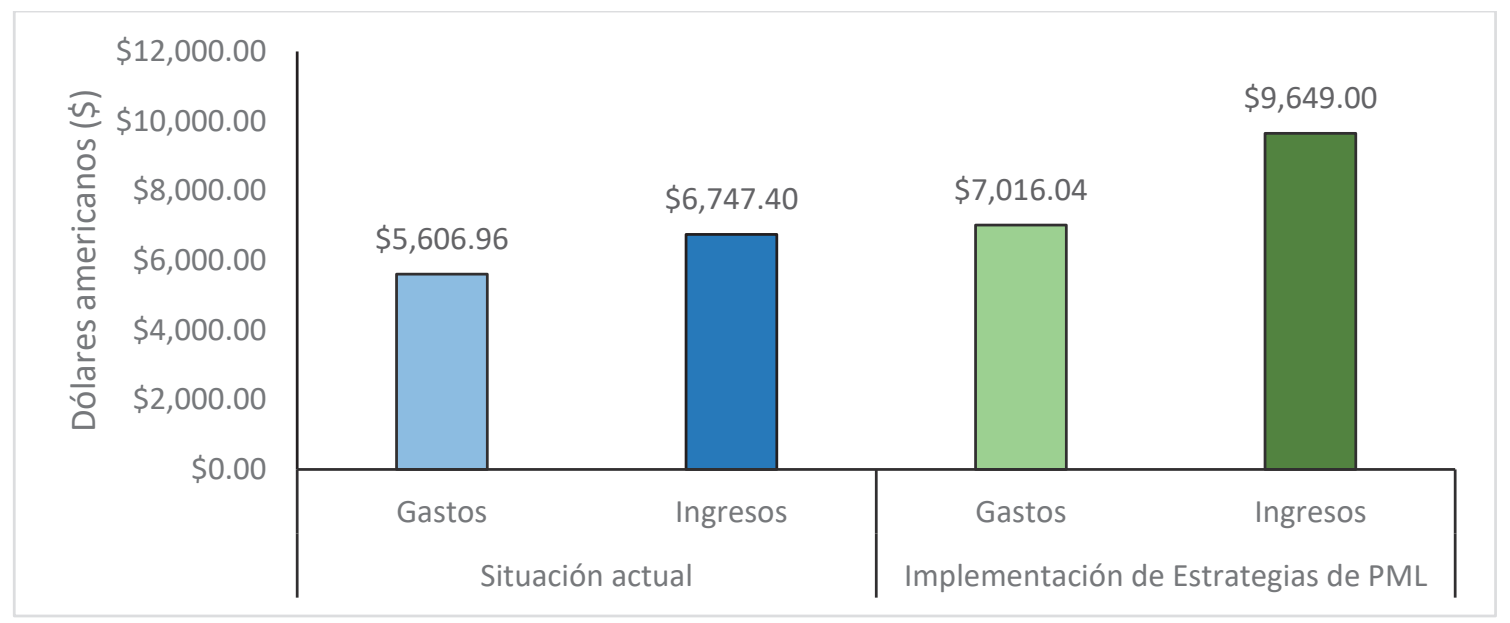

Figura 3. Comparación económica de la situación actual con la implementación de las estrategias de PML.

Fuente: Elaboración propia.

Tabla 5. Eco-indicadores por kg de masa de cacao, situación actual e implementación de estrategias de PML.

\begin{tabular}{|lccc|}
\hline \multicolumn{1}{|c}{ Eco-indicador } & Unidad & Valor \\
\hline Energía Eléctrica & $\mathrm{kW} / \mathrm{mes} /$ & 3.64 & Estrategias de PML \\
Consumo de Agua & $\mathrm{m}^{3}$ & 0.55 & 0.01 \\
Consumo de gas & $\mathrm{kg}$ & 0.05 & 0.64 \\
Producción de Cascarilla & $\mathrm{kg}$ & 0.11 & 0.11 \\
Producción de mucilago de cacao & $\mathrm{L}$ & 0.07 & 0.00 \\
\hline
\end{tabular}

Fuente: Elaboración propia. 
Los eco-indicadores analizados, muestran cambios luego de la implementación de las opciones de $\mathrm{PML}$, en este caso, se disminuye la generación de residuos tales como: mucílago y cascarilla del grano de cacao, los mismos que son aprovechados en un $100 \%$ para la elaboración de nuevos subproductos, entre estos un herbicida natural a partir de mucílago de cacao, y té de cascarilla en combinación con guayusa. Esta reducción de los residuos generados del proceso significa en términos ambientales una disminución de la contaminación, puesto que la problemática de la generación de residuos fue de las principales identificadas en el diagnóstico. En el caso del mucilago también repercute positivamente en las aguas residuales, que podrán disminuir las cargas orgánicas al no ser vertido incontroladamente cada vez que se realiza la fermentación del cacao. Por consecuente a esto, se obtiene que al incrementar la producción de la empresa también incrementa el índice de consumo de recursos como energía, agua y gas utilizados en su fabricación, aunque las opciones contribuirán a producir otros bienes e ingresos para ayudar a la economía de la empresa. En resumen, las estrategias de PML, inciden en mejorar los procesos y controlar la contaminación de la producción de cacao fino de aroma de la Asociación.

\section{CONCLUSIONES}

Los principales problemas detectados en el diagnóstico realizado en la asociación de productores de cacao fino de aroma Tsatsayaku, son la generación de mucílago y de cascarilla de cacao. Las alternativas de PML consideradas fueron la elaboración de un herbicida del mucílago de cacao y un té de cascarilla en combinación con guayusa. De acuerdo con la evaluación técnica económica y ambiental realizada estas opciones de producción más limpia presenta resultados positivos puesto que según la relación beneficio/costo generará un aumento de $\$ 0.18$ más de ganancias que en el estado actual. Además, en el periodo de tres años tendrá un valor actual neto de $\$ 36,001.37$ y una tasa interna de retorno de $249.29 \%$. Adicionalmente, con la aplicación de las opciones se reducirá la cantidad generada de residuos sólidos (mucílago y cascarilla) durante el proceso productivo y las opciones de PML permitirán la diversificación por la elaboración de nuevos productos. No obstante, es recomendable la búsqueda de nuevas alternativas que optimicen el uso de recursos y se recomienda considerar las buenas prácticas de manufactura al igual que optimizar el proceso de producción de pasta de cacao en la asociación Tsatsayaku.

\section{REFERENCIAS BIBLIOGRÁFICAS}

[5] Arteaga-Pérez, L. E., Segura, C., y Santana, K. D. (2016). Procesos de torrefacción para valorización de residuos lignocelulósicos. Análisis de posibles tecnologías de aplicación en Sudamérica. Afinidad, 73(573), 60-68.

[6] Arteaga, Y. (2013). Estudio del desperdicio del mucilago de cacao en el cantón Naranjal (Provincia del Guayas). ECA sinergia, 4(1), 49-59.

[7] Asamblea-Constituyente (20 de Octubre del 2008). Constitución de la República del Ecuador, Registro Oficial No. 449.

[8] Asamblea-Nacional (12 de abril de 2017). Código Orgánico del Ambiente, Registro Oficial $\mathrm{N}^{\circ} 983$.

[9] Blackman, A., y Kildegaard, A. (2010). Clean technological change in developing-country industrial clusters: Mexican leather tanning. Environ Econ Policy Stud, 12. doi:https://doi. org/10.1007/s10018-010-0164-7

[10] Caiza, D., Chimbo, A., Sarduy-Pereira, L. B., Pisco, W., y Diéguez-Santana, K. (2018). Propuesta de producción más limpia en el proceso de elaboración de abonos orgánicos con desechos del camal, realizado en el relleno sanitario del cantón Baños de Agua Santa, provincia de Tungurahua. Revista Observatorio de la Economía Latinoamericana, 6.

[11] Cárdenas, E. V., Maldonado, J. M., Valdez, R. A., Sarduy-Pereira, L. B., y Diéguez-Santana, K. (2019). La producción más limpia en el sector porcino. Una experiencia desde la Amazonia Ecuatoriana. Anales Científicos, 80(1), 76-91. doi:http://dx.doi.org/10.21704/ ac.v80i1.1288

[12] Castro, D., Oliveira, Â. M., Tortorella, G. L., Zandonai, G. A., y Soares, M. E. (2017). Improvements in the processing of agricultural commodities: The case of cocoa liquor. Dyna, 84(201), 117-122. doi:http://dx.doi. org/10.15446/dyna.v84n201.56672

[13] CEER. (2016). Centro de Eficiencia de Recursos y Producción Más Limpia en Ecuador. Recuperado de https://www.industrias. gob.ec/centro-de-eficiencia-de-recursos-yproduccion-mas-limpia/

[14] CEER. (2019). Guía de producción más limpia: Centro Ecuatoriano de Eficiencia de Recursos y Producción Más limpia CEER 
[15] CPTS. (2005). Guía Técnica General de Producción más Limpia. La Paz, Bolivia: Centro de Promoción de Tecnologías Sostenibles, CPTS, USAID.

[16] Denham, F. C., Howieson, Janet R., Solah, V. A., y Biswas, W. K. (2015). Environmental supply chain management in the seafood industry: past, present and future approaches. Journal of Cleaner Production, 90, 82-90. doi:https:// doi.org/10.1016/j.jclepro.2014.11.079

[17] Diéguez-Santana, K., Arteaga-Pérez, L. E., Casas Ledon, Y., y Rodríguez Rico, I. L. (2013). Análisis de ciclo de vida y caracterización ambiental en una industria alimenticia. Revista Centro Azúcar, 40, 52-58.

[18] Diéguez-Santana, K., Casas-Ledón, Y., Loureiro-Salabarria, J. A., Pérez-Martínez, A., y Arteaga-Pérez, L. E. (2020). A life cycle assessment of bread production: A Cuban case study. Journal of Environmental Accounting and Management, 8(2), 125-137. doi:https:// doi.org/10.5890/JEAM.2020.06.002

[19] Filho, J. C., Nunhes, T. V., y Oliveira, O. J. (2019). Guidelines for cleaner production implementation and management in the plastic footwear industry. Journal of Cleaner Production, 232, 822-838. doi:https://doi. org/10.1016/j.jclepro.2019.05.343

[20] Fritzen-Gomes, B. M. (2020). Cleaner Production and Technologies. In W. Leal Filho, A. M. Azul, L. Brandli, P. G. Özuyar, y T. Wall (Eds.), Responsible Consumption and Production (pp. 90-98). Cham: Springer International Publishing.

[21] Guallo-Aguinda, N. G., Sarduy-Pereira, L. B., Orozco-Crespo, E., y Diéguez-Santana, K. (2020). Las producciones más limpias en el sector textil manufacturero. Un caso de estudio en Tena, Napo, Ecuador. Mikarimin. Revista Científica Multidisciplinaria, 6, 201-218.

[22] Konstantas, A., Jeswani, H. K., Stamford, L., y Azapagic, A. (2018). Environmental impacts of chocolate production and consumption in the UK. Food Research International, 106, 1012-1025. doi:https://doi.org/10.1016/j. foodres.2018.02.042

[23] Li, X., y Hamblin, D. (2016). Factors impacting on cleaner production: case studies of Chinese pharmaceutical manufacturers in Tianjin, China. J Clean Prod, 131. doi:https://doi.org/10.1016/j. jclepro.2016.05.066
[24] Llerena, W. T., Ah-Hen, K., y Lemus-Mondaca, R. (2017). Caracterización de una infusión de cascarilla de cacao (Theobroma cacao L., var. Arriba) con hierbas aromáticas. Agro sur, 45(3), 47-55. doi:https://doi.org/10.4206/ agrosur.2017.v45n3-07

[25] Luken, R., y Navratil, J. (2004). A programmatic review of UNIDO/UNEP national cleaner Production centres. J Clean Prod, 12 (3), 195-205. doi:https://doi.org/10.1016/S09596526(03)00102-1

[26] Luken, R., Van Berkel, R., Leuenberger, H., y Schwager, P. (2016). A 20-year retrospective of the united nations industrial development organization and united nations environment programme national cleaner production centres programme. J Clean Prod, 112, 1165-74. doi:https://doi.org/10.1016/j. jclepro.2015.07.142

[27] MAG. (2019). Sitema de información pública agropecuaria. Productos agropecuarios. Principales productos agrícolas. Cacao. Recuperado de http://sipa.agricultura.gob.ec/ index.php/comercio-exterior

[28] Mancheno, N. R. (2018). Plan de marketing para la asociación de productores de cacao de fino aroma Ttsatsayaku) del canton Carlos Julio Arosemena Tola, provincia Napo, para el año 2017. (Tesis previa a optar el Grado de Ingeniera Comercial), Universidad Nacional de Loja, Loja, Ecuador.

[29] Matos, L. M., Anholon, R., da Silva, D., Cooper Ordoñez, R. E., Gonçalves Quelhas, O. L., Filho, W. L., y de Santa-Eulalia, L. A. (2018). Implementation of cleaner production: A tenyear retrospective on benefits and difficulties found. Journal of Cleaner Production, 187, 409-420. doi:https://doi.org/10.1016/j. jclepro.2018.03.181

[30] Neira, D. P. (2016). Energy sustainability of Ecuadorian cacao export and its contribution to climate change. A case study through product life cycle assessment. Journal of Cleaner Production, 112, 2560-2568. doi:https://doi. org/10.1016/j.jclepro.2015.11.003

[31] Okiyama, D. C. G., Navarro, S. L. B., y Rodrigues, C. E. C. (2017). Cocoa shell and its compounds: Applications in the food industry. Trends in Food Science \& Technology, 63, 103-112. doi:https://doi.org/10.1016/j. tifs.2017.03.007 
[32] Padda, I. U. H., y Asim, M. (2019). What determines compliance with cleaner production? An appraisal of the tanning industry in Sialkot, Pakistan. Environmental Science and Pollution Research, 26(2), 1733-1750. doi:https://doi. org/10.1007/s11356-018-3717-0

[33] Rahim, R., y Raman, A. A. (2015). Cleaner production implementation in a fruit juice production plant. Journal of Cleaner Production, 101, 215-221. doi:10.1016/j. jclepro.2015.03.065

[34] Recanati, F., Marveggio, D., y Dotelli, G. (2018). From beans to bar: A life cycle assessment towards sustainable chocolate supply chain. Science of The Total Environment, 613-614, 1013-1023. doi:https://doi.org/10.1016/j. scitotenv.2017.09.187

[35] Roy, C. (2012). A Study on Environmental Compliance of Indian Leather Industry \& its Far-reaching Impact on Leather Exports. Foreign Trade Review, 47(2), 3-36. doi:https:// doi.org/10.1177/0015732515120201

[36] Sanchez, D. M., Perez, W. R., Rojas, D. F. C., y Trujillo, E. T. (2019). Respuesta agronómica de mucilago de cacao (Theobroma cacao L.) en cultivo de maíz (Zea mays L.). Ciencia en desarrollo, 10(2), 43-58. doi:https://doi. org/10.19053/01217488.v10.n2.2019.7958
[37] Soto-Cabrera, A. I., Panimboza-Ojeda, A. P., Ilibay-Granda, C. G., Valverde-Lara, C. R., y Diéguez-Santana, K. (2020). Impacto ambiental de la operación del Centro de faenamiento de la ciudad de Puyo, Pastaza, Ecuador. Prospectiva, 18(1), 60-68. doi:https:// doi.org/10.15665/rp.v18i1.2101

[38] UNEP. (1990). Resource efficiency and cleaner production. Recuperado de http://www.unep. org/recp/

[39] UNEP. (2015). Sustainable Consumption and Production: A Handbook for Policymakers: United Nations Environment Programme.

[40] Vásquez, Z. S., de Carvalho Neto, D. P., Pereira, G. V. M., Vandenberghe, L. P. S., de Oliveira, P. Z., Tiburcio, P. B., . . . Soccol, C. R. (2019). Biotechnological approaches for cocoa waste management: A review. Waste Management, 90, 72-83. doi:https://doi. org/10.1016/j.wasman.2019.04.030 